\title{
Chronic Use of Azithromycin Might Explain the Low Prevalence of COVID-19 in Cystic Fibrosis Patients
}

\section{Sara Galimberti*}

Department of Clinical and Experimental Medicine, University of Pisa, Pisa, Italy

*Corresponding author: Prof. Sara Galimberti, Department of Clinical and Experimental Medicine, University of Pisa, Pisa, Italy

Severe Acute Respiratory Syndrome - Coronavirus 2 (SARS-CoV-2) is the virus responsible for the pandemic that in the first months of 2020 caused worldwide more than 8 millions of infected subjects and 436,000 deaths.

It has been reported that only few individuals affected by cystic fibrosis (CF) developed the clinical manifestations of the often dramatic SARS-CoV-2 related disease, now known as Coronavirus Disease 19 (COVID-19).

In Europe, (data updated at 10 June 2020), only 90 cases of COVID-19 in CF patients have been reported, with 6 subjects requiring admission into intensive care unit (ICU) and 3 deaths. Clinical data are now available for 42 hospitalized patients: 32 of them presented with "mild", 7 with "severe" and 3 with "critical" COVID-19. In more than $70 \%$ of CF patients the SARS-CoV- 2 infection impaired the pulmonary function, while $42 \%$ of them remained totally asymptomatic (https://www. ecfs.eu/news/covid-cf-project-europe).

From the epidemiological point of view, these numbers seem to be very impressive, if we consider that in Europe it has been estimated to live 48,000 CF patients (https://www.ecfs.eu/sites/default/files/general-content-images/working-groups/ecfs-patient-registry/ECFSPR_Report2017_v1.3.pdf) and that the rate of infection by Coronavirus ranged from 29/100,000 (in Greece) to $393 / 100,000$ (in Italy) and 522/100,000 in Spain (https://www.ecdc.europa.eu/en/cases-2019ncov-eueea).

Also the outcome of COVID-19 in CF population seems to be favorable, if we consider that in the general population $10 \%$ of infected individuals during pandemic required hospitalization in ICU [1].

Dr. Colombo reported that, at the $31^{\text {st }}$ March 2020, in Lombardia (Italy) Coronavirus was documented in $10 \mathrm{CF}$ patients out of 42,161 infected people, so confirming the low rate of infection or at least of COVID-19 clinical manifestations in CF population [2]. Different possible explanations for this phenomenon have been hypothesized: 1) The younger age of CF patients, 2) The immediate minimization of social contacts, and 3) The probable protective role of some drugs that CF patients commonly receive, such as azithromycin. This third option might be particularly relevant, considering that it is well known that azithromycin is a "senolytic" drug and that the senescence has been reported to be fundamental in COVID-19 also [3]. In CF, azithromycin is usually employed because its ability of increasing FEV1 [4] and of playing an anti-inflammatory action [5].

In COVID-19, its role has been recently debated: indeed, it has been initially reported that this antibiotics induced a rapid viral load negativization when used in combination with hydroxychloroquine [6], but a recent review showed conflicting results, either in vitro or in vivo, even if in a small series a superior viral clearance in patients treated with azithromycin and hydroxychloroquine, compared with hydroxychloroquine alone, has been reported [7].

During the first phase of attack, SARS-CoV-2 seems to fight the host both by blocking autophagy, the first line of host anti-viral defense [8], and by increasing the cellular senescence [3]. During infection, one of viral proteins, the open reading frame $9 b(O r f-9 b)$, goes to

Citation: Galimberti S (2020) Chronic Use of Azithromycin Might Explain the Low Prevalence of COVID-19 in Cystic Fibrosis Patients. Int Arch Clin Pharmacol 6:023. doi.org/10.23937/25723987.1510023

Received: May 23, 2019: Accepted: June 27, 2019: Published: June 29, 2020

Copyright: (c) 2020 Galimberti S. This is an open-access article distributed under the terms of the Creative Commons Attribution License, which permits unrestricted use, distribution, and reproduction in any medium, provided the original author and source are credited. 
localize itself inside the host cells mitochondria where it blocks the interferon-dependent anti-viral action by the proteasome-dependent degradation of the mitochondrial Dynamin-1 like (DRP1) protein. The consequences of this viral activity are several mitochondrial abnormalities and dysfunctions, such as hyperfusion, with the final acquisition for the infected host cells of the senescent phenotype. Once senescent, the infected cells start to widespread the new viruses by producing high amounts of extra-cellular vesicles, such as exosomes, that can reach different sites and further damage different tissues. At the same time, senescent cells overproduce pro-inflammatory cytokines, chemokines and growth factors, responsible for the COVID-19 onset [3]. Indeed, patients with COVID-19 show lymphopenia, elevated levels of pro-inflammatory cytokines (TNF alpha, IL2, IL6, IL17, macrophage inflammatory protein 1 alpha), atrophy of spleen and lung infiltration characterized by a high number of macrophages. This population would be responsible for the "primary" cytokine storm, whilst the "secondary" one might be provoked by the activated T lymphocytes [9].

Recently, our group reported the important role of neutrophils that would be able to form neutrophil extracellular traps (NETs) that may actively contribute to hyper-inflammation and thrombotic events that in COVID-19 are frequently reported [10]. Interestingly, it has been demonstrated that pre-incubation of neutrophils isolated from healthy donors with increasing concentrations of azithromycin significantly decreased the release of NETs [11]. Considering that in CF neutrophils can extrude large amounts of nuclear material through NETs into the airways of patients, so contributing to the lung damage [12], the "anti-NET" activity of azithromycin might once again make these antibiotics an effective bullet against COVID-19 and CF.

In conclusion, SARS-CoV-2, blocking autophagy (especially by using the $\mathrm{CD} 26$ receptor), and inducing high rate of senescence, is able to fast replicate before the onset of host's immune response onset. Azithromycin, that already plaid an anti-viral action against Zika and influenza H1N1 viruses [13], by its "senolytic" and "anti-NET" actions, might play a defensive role also against SARS-CoV-2, so probably at least in part contribute to explain the low incidence of COVID-19 in CF population.

\section{Declaration of Competing Interest}

I declare that I have no known competing financial interests or personal relationships that could have influenced what reported in this paper.

\section{Acknowledgements}

This manuscript is dedicated to G.T, L.T, G.T, O.T.

\section{References}

1. Remuzzi A, Remuzzi G (2020) COVID-19 and Italy: what next?. Lancet 395: 1225-1228.

2. Colombo C, Burgel PR, Gartner S, van Koningsbruggen-Rietschel S, Naehrlich L, et al. (2020) Impact of COVID-19 on people with cystic fibrosis. Lancet Respir Med 8: e35-e36.

3. Malavolta M, Giacconi R, Brunetti D, Provinciali M, Maggi $F$ (2020) Exploring the Relevance of Senotherapeutics for the Current SARS-CoV-2 Emergency and Similar Future Global Health Threats. Cells 9: 909.

4. Lisa Saiman, Bruce C Marshall, Nicole Mayer-Hamblett, Jane L Burns, Alexandra L Quittner, et al. (2003) Azithromycin in patients with cystic fibrosis chronically infected with Pseudomonas aeruginosa: A randomized controlled trial. JAMA 290: 1749-1756.

5. Cigana C, Nicolis E, Pasetto M, Assael BM, Melotti $P$ (2006) Anti-inflammatory effects of azithromycin in cystic fibrosis airway epithelial cells. Biochem Biophys Res Commun 350: 977-982.

6. Okour M, Al-Kofahi M, Austin D (2020) Hydroxychloroquine and azithromycin as potential treatments for COVID-19; clinical status impacts the outcome. Journal of Pharmacokinetics and Pharmacodynamics 47: 187-188.

7. Gbinigie K, Frie K (2020) Should azithromycin be used to treat COVID-19? A rapid review. BJGP Open bjgpopen20X101094.

8. Sara Galimberti, Susanna Grassi, Claudia Baratè, Francesca Guerrini, Elena Ciabatti, et al. (2018) The Polycomb BMI1 protein is co-expressed with CD26+ in leukemic stem cells of chronic myeloid leukemia. Front Oncol 8: 555.

9. Misra DP, Agarwal V, Gasparyan AY, Zimba O (2020) Rheumatologists' perspective on coronavirus disease 19 (COVID-19) and potential therapeutic targets. Clin Rheumatol 39: 2055-2062.

10. Francesco Ferro, Elena Elefante, Ilaria Puxeddu, Chiara Baldini, Elena Bartoloni, et al. (2020) COVID-19: The new challenge for rheumatologists. First update. Clin Exp Rheumatol 38: 373-382.

11. Bystrzycka W, Manda-Handzlik A, Sieczkowska S, Moskalik A, Demkow U, et al. (2017) Azithromycin and chloramphenicol diminish neutrophil extracellular traps (NETs) release. Int J Mol Sci 18: 2666.

12. Martínez-Alemán SR, Campos-García L, Palma-Nicolas JP, Hernández-Bello R, González GM, et al. (2017) Understanding the Entanglement: Neutrophil Extracellular Traps (NETs) in cystic fibrosis. Front Cell Infect Microbiol 7: 104.

13. Tran DH, Sugamata R, Hirose T, Suzuki S, Noguchi $Y$, et al. (2019) Azithromycin, a 15-membered macrolide antibiotic, inhibits influenza $A(\mathrm{H} 1 \mathrm{~N} 1)$ pdm09 virus infection by interfering with virus internalization process. J Antibiot 72: 759-768.

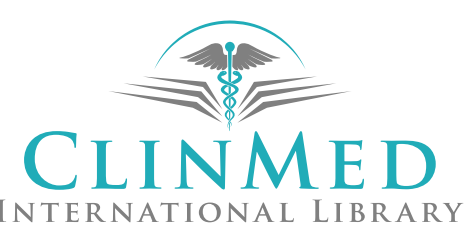

